5th International EEIGM/AMASE/FORGEMAT Conference on Advanced Materials Research IOP Publishing IOP Conf. Series: Materials Science and Engineering 5 (2009) 012020 doi:10.1088/1757-899X/5/1/012020

\title{
Effect of thermal aging and fatigue on failure resistance of aerospace composite materials
}

\author{
K Giannadakis and J Varna \\ Dept of Applied Physics and Mechanical Engineering \\ Division of Polymer Engineering, Lulea University of Technology,SE 97187, Sweden \\ E-mail: Janis.Varna@ltu.se
}

\begin{abstract}
Exposing a laminate structure to thermal cycles and to temperatures close to the curing temperature, followed by mechanical fatigue, cause interlaminar and intralaminar cracks leading to degradation of the mechanical properties. The effect of thermal and mechanical fatigue, as well as thermal aging, on carbon fiber composite laminate structures is under study in the present paper.
\end{abstract}

\section{Introduction}

The increasing needs of aeronautical applications have turned interest to composite materials. This is due to high mechanical and thermal properties, at a lower weight, longer lifetime and reusability of composites.

Some of the issues that these materials face are caused by exposure to high temperatures, large temperature variations $\left(-50\right.$ to $\left.+40{ }^{\circ} \mathrm{C}\right)$ and mechanical fatigue due to pressure difference and vibrations of various structural components during a flight. The prementioned are main damage sources, in terms of crack initiation and propagation and they are responsible for the material's degradation [1].

In the present paper the effect of thermal fatigue and thermal aging, on mechanical performance of carbon fiber laminate composites in tensile quasi-static loading and in mechanical fatigue have been studied. The main goal is to quantify the effect of the above degradation sources in terms of reduced resistance to matrix crack initiation and propagation in laminated structures. The effect of crack formation in composites has also been investigated in $[2,3]$.

Multiple matrix cracks, called also intralaminar cracks, are observable in layer with off-axis orientation with respect to the main loading direction. These cracks usually initiate in weekest positions or in stress concentration areas, they cover the whole layer thickness and grow parallel to fibers in the layer. The statistical transverse failure properties in terms of Weibull distribution in the layer can be obtained from experimental data on cross-ply laminates expressing the linear crack density as a function of the applied strain which is translated to the thermo-mechanical stress in the layer. Weibull parameters are compared for the "healthy" untreated composite and the material after certain thermal treatment.

Two tests were performed on treated and untreated cross-ply carbon fiber laminate specimens. Quasistatic mechanical loading and unloading in steps with increasing maximum strain was performed, measuring the number of cracks after every step. The mechanical fatigue consisted of a cyclic tension-tension load application and the relation between the number of cycles and the number 
5th International EEIGM/AMASE/FORGEMAT Conference on Advanced Materials Research IOP Publishing IOP Conf. Series: Materials Science and Engineering 5 (2009) 012020 doi:10.1088/1757-899X/5/1/012020

of formed cracks was investigated. The effect of mechanical fatigue has also been investigated in $[4,5]$.

Efforts were made to understand if the multiple damage in tests is initiation or propagation governed. In the first case the stress to initiate defect large enough to propagate requires high stress which exceeds the applied stress level needed for crack propagation. As a consequence the crack propagation is unstable. If the initiation stress is low the cracking is propagation governed and can be stable as well as unstable. However, in the latter case the fracture mechanics can be used to describe observations and one of the expected phenomena is dependence of the multiple cracking characteristics on the cracked ply thickness. Thermal treatment can differently affect the degradation of initiation and propagation resistance characteristics. The effect of accelerating material degradation due to exposure to high temperatures has also been described in [6].

In order to estimate the effect of thermal aging, the material was exposed to temperaratures close to the curing temperature for two different time intervals and the same cracking characteristics, related to matrix degradation, were studied.

\section{Mechanical Fatigue}

The materials involved in this study are two carbon fibers (CF) composites with RTM6-resin for the first, and BMI-resin for the second one. Two different layouts have been studied: $[0 / 90 / 0 / 90]_{\mathrm{s}}$ and $\left[0_{2} / 90_{2}\right]_{\mathrm{s}}$. The thickness of one layer was about $0.53 \mathrm{~mm}$, with typical sample's length and width, $200 \mathrm{~mm}$ and $15 \mathrm{~mm}$ respectively. A series of tension-tension fatigue loading experiments were carried out on all samples in order to study the development of transverse intralaminar cracks. The most important parameters for this kind of testing are the preloading (or pre-strain), the frequency, the ratio ( $R=\frac{\sigma_{\min }}{\sigma_{\max }}$ ) between the minimum and maximum stress value and the number of cycles. For the case of CF/BMI the maximum stress was approximately $115 \mathrm{MPa}$, as for the case of CF/RTM6 it was 130MPa. The set of parameters used is described on Table 1.

Table 1: Fatigue test parameters

\begin{tabular}{|l|l|l|l|l|l|}
\hline Frequency & \multicolumn{4}{l|}{$10 \mathrm{~Hz}$} \\
\hline Pre-load & \multicolumn{4}{l|}{$0.4 \%$ strain } \\
\hline Ratio & 0.1 & \multicolumn{4}{|l|}{} \\
\hline Number of cycles & 1 & 50 & 500 & 5000 & 20000 \\
\hline
\end{tabular}


5th International EEIGM/AMASE/FORGEMAT Conference on Advanced Materials Research IOP Publishing IOP Conf. Series: Materials Science and Engineering 5 (2009) 012020ｄoi:10.1088/1757-899X/5/1/012020

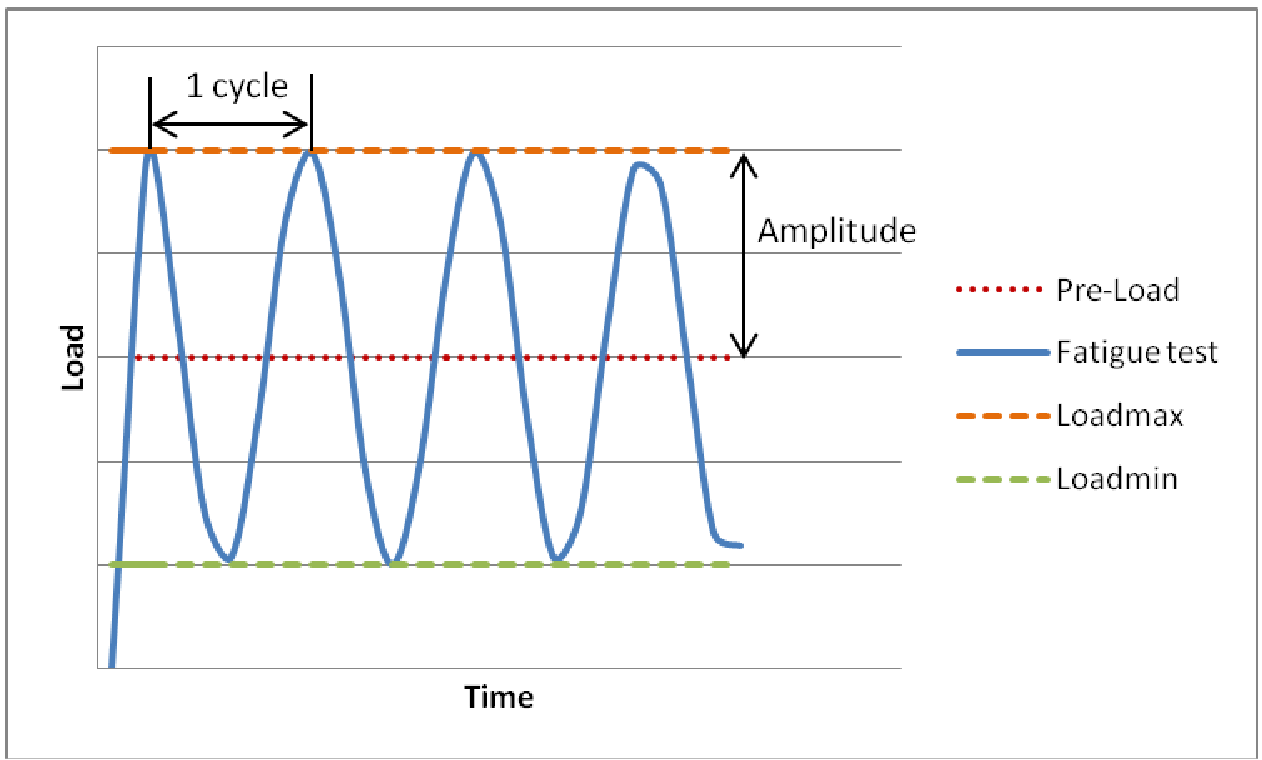

Figure 1: Mechanical Fatigue Test Parameters

For each number of cycles the respective number of cracks was counted and the crack density was calculated as the number of crack per unit length in the layer. The results for individual specimens are presented on Figures 2 and 3 for CF/RTM6 and CF/BMI. The results in Figures 2 and 3 correspond to cracks in the thinnest $90^{\circ}$ layers of two $[0 / 90 / 0 / 90]_{\mathrm{s}}$ specimens and to cracks in two $\left[0 / 90_{2}\right]_{\mathrm{s}}$ specimens with thick $90^{\circ}$ layer.

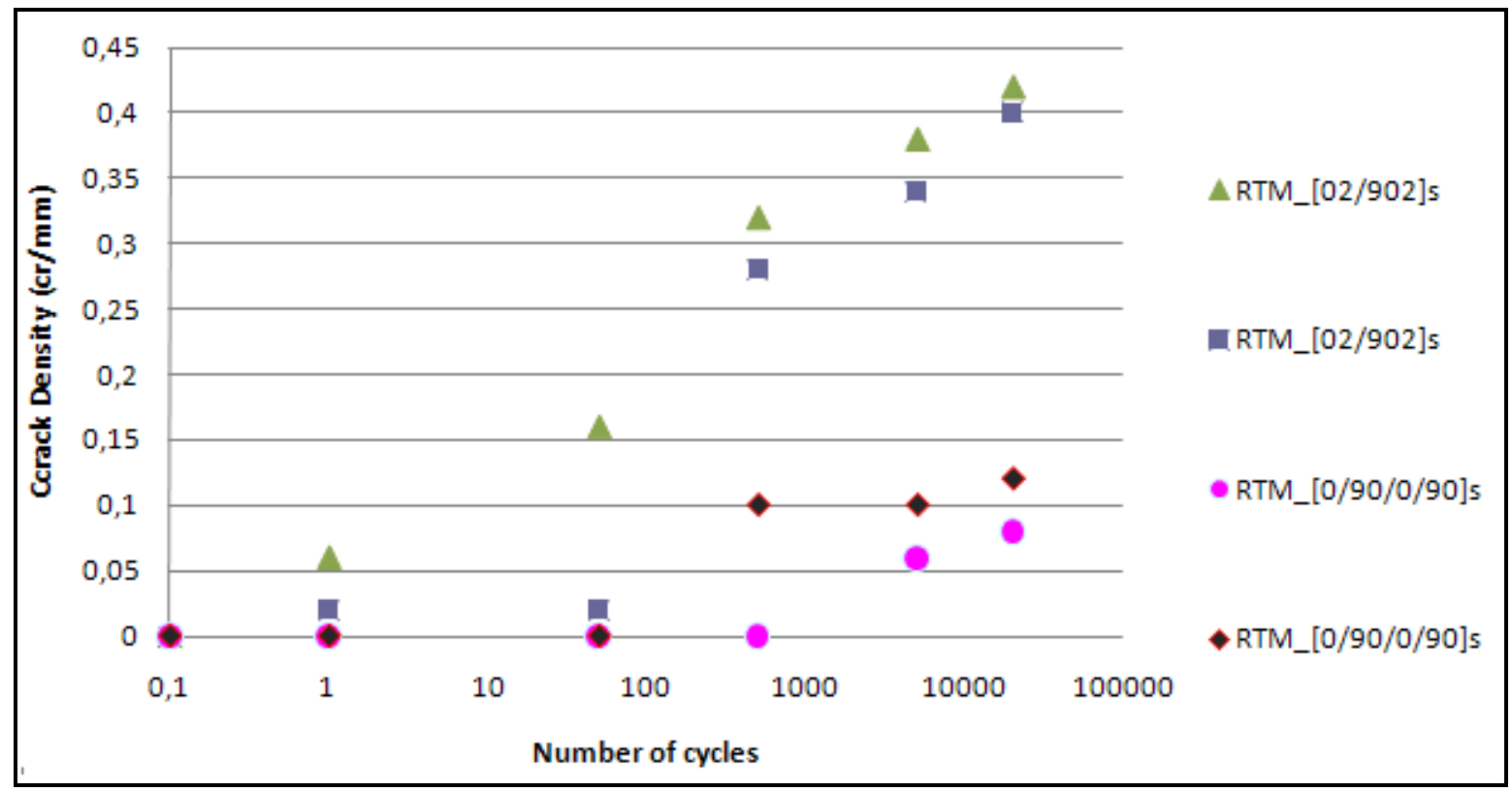

Figure 2: Crack Density comparison between layouts for RTM6 composites 
5th International EEIGM/AMASE/FORGEMAT Conference on Advanced Materials Research IOP Publishing IOP Conf. Series: Materials Science and Engineering 5 (2009) 012020ｄoi:10.1088/1757-899X/5/1/012020

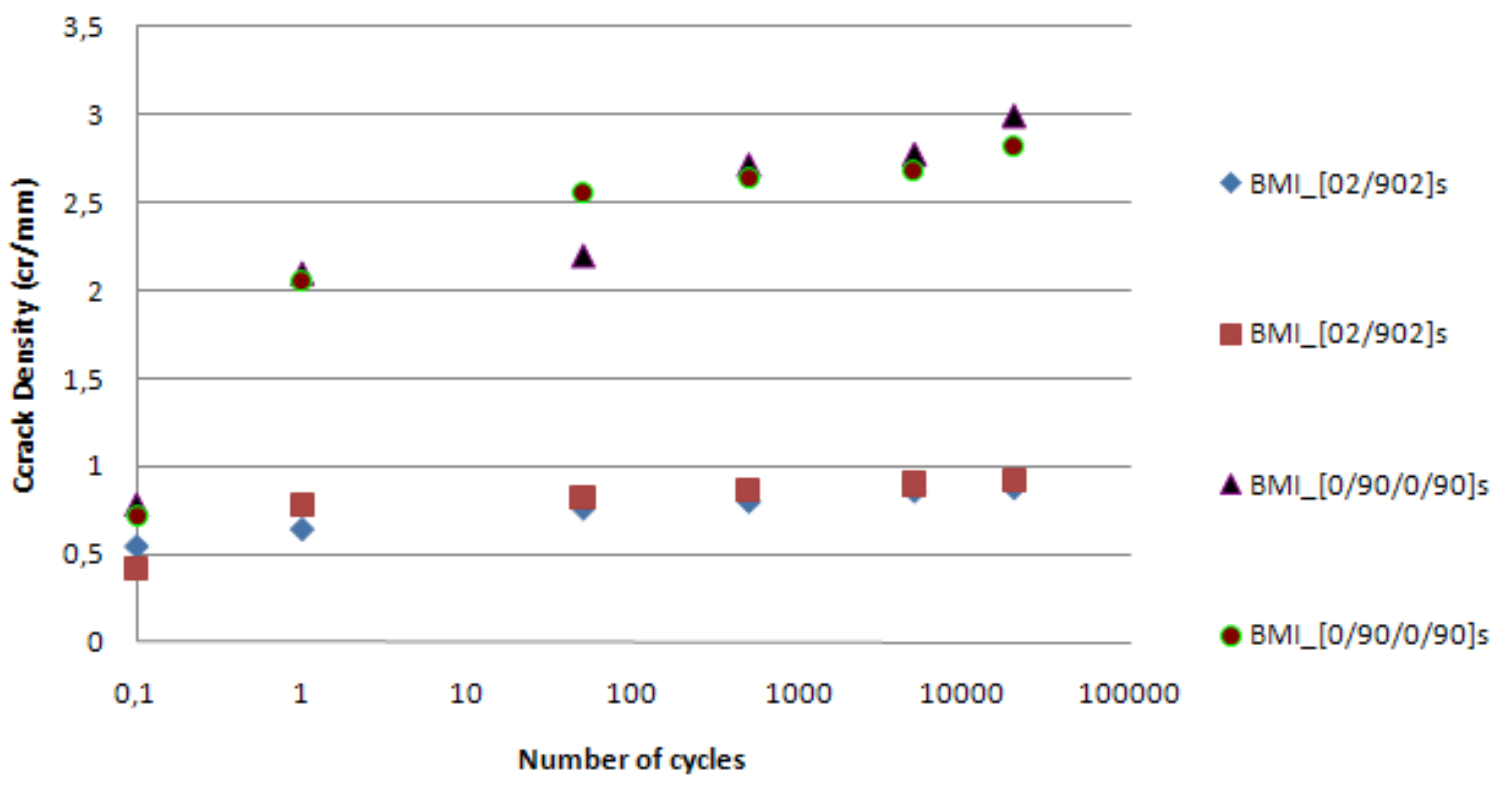

Figure 3: Crack Density comparison between layouts for BMI composites

The main conclusion is that the actual stacking sequence is of great importance, since for both materials the crack density for four continuous $90^{\circ}$ plies is approximately twice as high as for two continuous plies. In addition, there is a big difference between BMI and RTM6 composites in terms of damage resistance; BMI material is much less damage resistant than RTM6.

\section{Thermal Fatigue}

The thermal fatigue and degradation effects are studied on CF epoxy [0/90] cross-ply laminates, manufactured using vacuum bag and hot press at $120^{\circ} \mathrm{C}$ for one hour. To prove that the composite undergoes degradation of tensile transverse strength and Mode I fracture toughness, it was subjected to thermal cycling between $+100^{\circ} \mathrm{C}$ and $-100^{\circ} \mathrm{C}$. It was expected that the failure resistance of the composite will be degraded due to high temperature difference, but also some transverse and longitudinal cracks will develop, as a result of cyclic fatigue due to high thermal stresses caused by low temperatures.

Finally, all samples were loaded in tension up to certain strain level and unloaded to count the number of cracks by means of optical microscopy. In this way the crack density in the $90^{\circ}$-layer was determined after each such a step. Then the next loading-unloading step with higher maximum strain was performed and cracks counted again. The focus was on damage initiation and, since thick $90^{\circ}$ layers $(0.3 \mathrm{~mm}$ in thickness) were used, the propagation stress was expected to be lower than the initiation stress thus simplifying optical microscopy studies.

The crack density versus applied strain depends on the strength distribution in the cracked layer and on the interaction between cracks, which starts to be significant at high crack densities and may be accounted for as described in [7]. These curves were used to determine statistical transverse strength distribution in the ply as dependent on the thermal treatment. 
5th International EEIGM/AMASE/FORGEMAT Conference on Advanced Materials Research IOP Publishing IOP Conf. Series: Materials Science and Engineering 5 (2009) 012020 doi:10.1088/1757-899X/5/1/012020

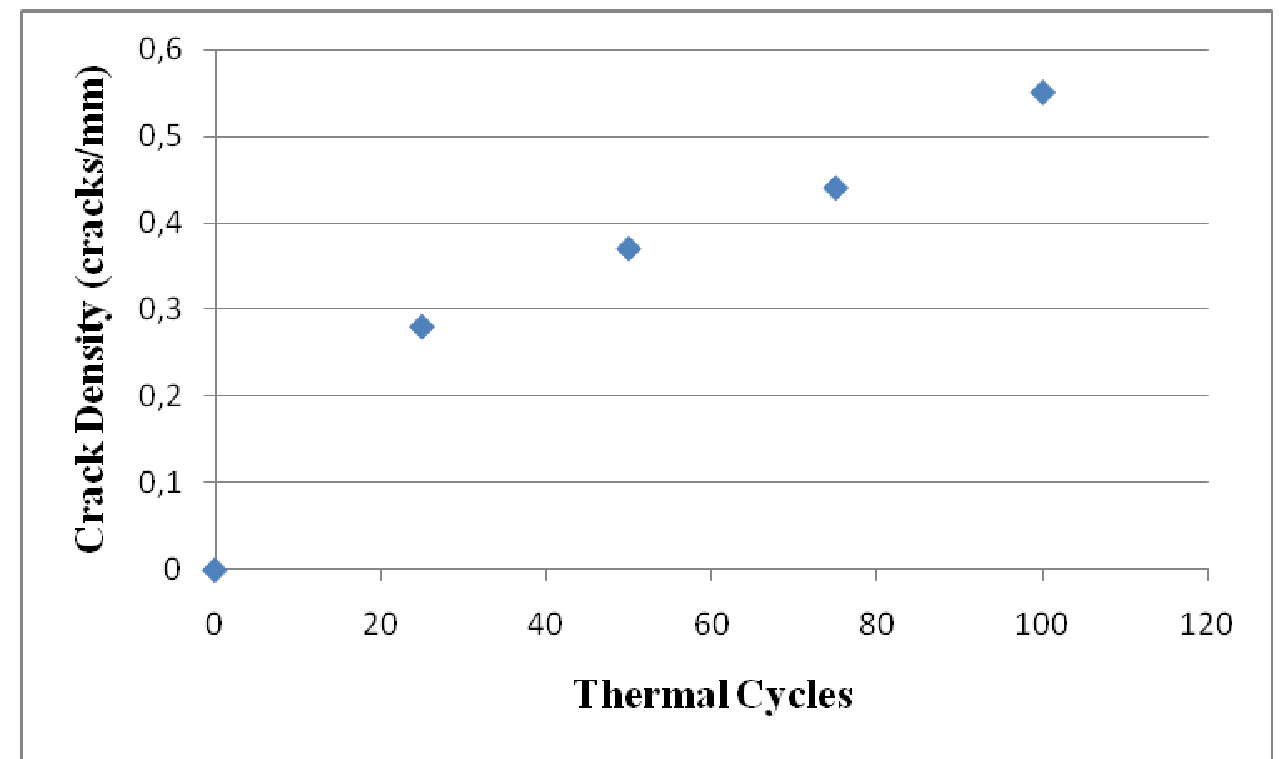

Figure 4: Dependence of Crack Density on the number of thermal cycles

In this procedure the thermal and curing stresses were added to transverse mechanical stress in the $90^{\circ}$-layer calculated using laminate theory from know applied mechanical strain. The thermal and curing stresses were obtained measuring temperature dependence of a curvature of an unsymmetric $\left(0^{\circ} / 90^{\circ}\right)$ laminate shown in Figures 5 and 6. 
5th International EEIGM/AMASE/FORGEMAT Conference on Advanced Materials Research IOP Publishing IOP Conf. Series: Materials Science and Engineering 5 (2009) 012020ｄoi:10.1088/1757-899X/5/1/012020

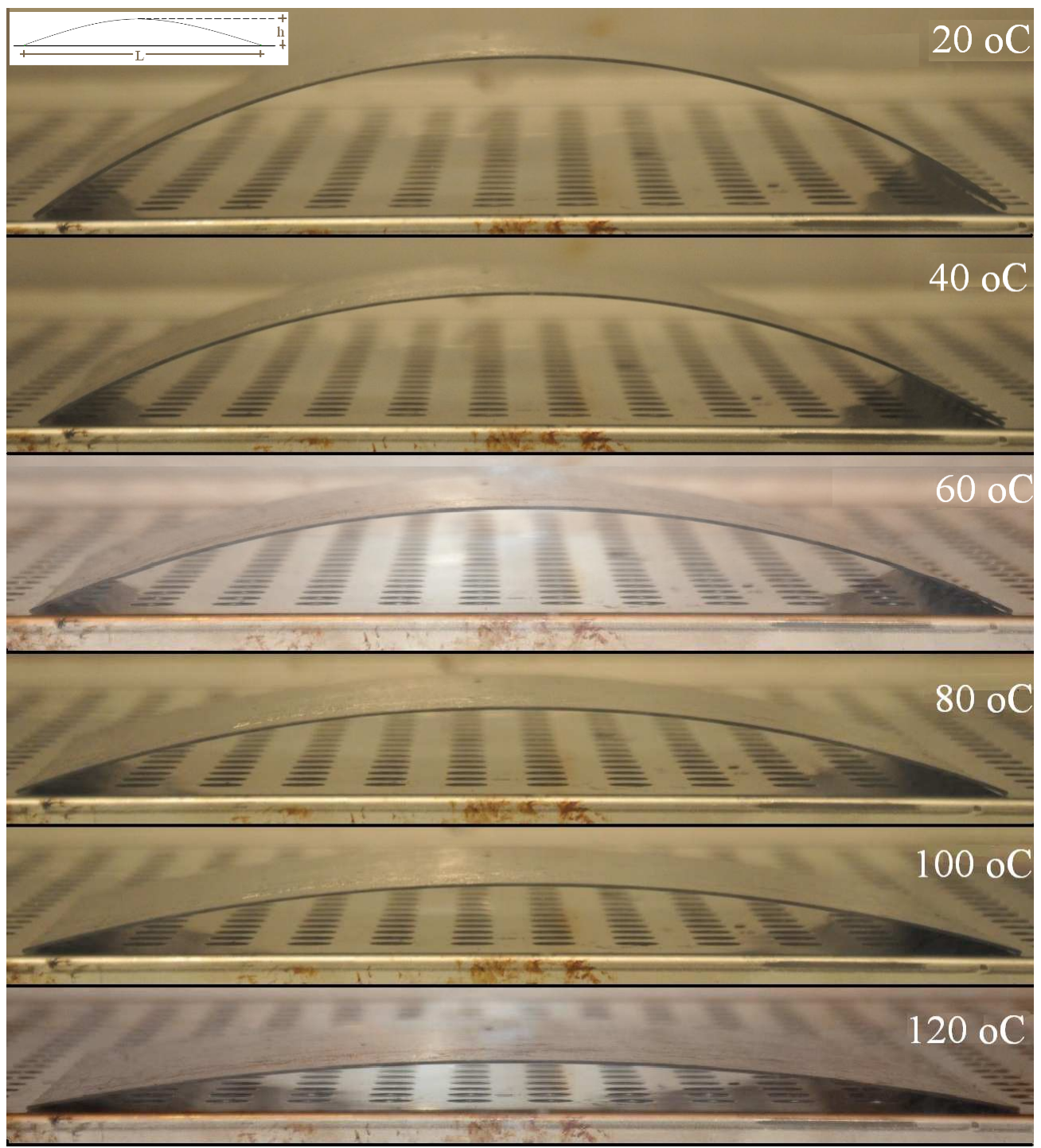

Figure 5: Curvature dependence on the temperature

The curing temperature of the composite plate was $120^{\circ} \mathrm{C}$. At this temperature, one would expect to have stress free condition. However, the temperature related variation of the curvature in Figure 6 shows that curvature is not zero at the manufacturing temperature, but at $235^{\circ} \mathrm{C}$. This is due to the matrix shrinkage and other chemical phenomena occurring during the curing process leading to composite shrinkage in the transverse direction. By extrapolating this line until it crosses the temperature axis, a "modified stress free temperature" can be obtained. This temperature can be used to include both thermal effects and the effect of chemical shrinkage during curing. The suggested use of the thermal analogy is very convenient. 
5th International EEIGM/AMASE/FORGEMAT Conference on Advanced Materials Research IOP Publishing IOP Conf. Series: Materials Science and Engineering 5 (2009) 012020ｄoi:10.1088/1757-899X/5/1/012020

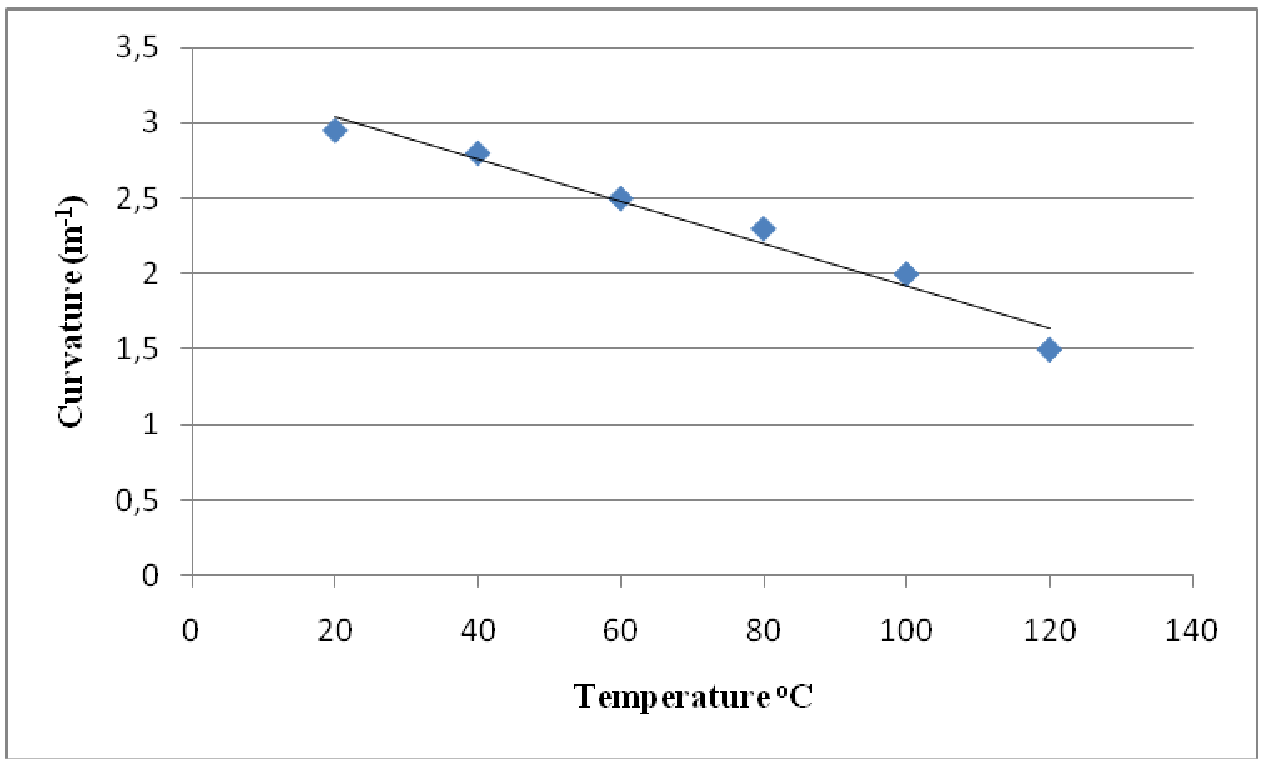

Figure 6: Curvature Dependence on the temperature

It has to be noted that many secondary damage modes were observed at specimen edges subjected to fatigue. The most typical are local delaminations and horizontal cracks. Specimens were cut in longitudinal directions (to see damage propagation through the width) and cracks on the new surfaces (middle of the specimen) were analyzed. Whereas intralaminar crack density was practically the same as on the edge, there were no local delaminations and other secondary damage modes detected in the middle of laminate. Thus for this material the specimen edge is representative for intralaminar cracking but not for other damage modes.

\section{Thermal aging effect}

Another parameter, responsible for mechanical properties degradation is the thermal aging. The material is exposed to temperatures close to the manufacturing temperature over a time interval. For short time intervals this thermal treatment can produce a material with superior mechanical properties, when compared to the initial, due to post curing phenomena. However, for longer time intervals the mechanical properties decrease primarily as a result of matrix degradation. This case study involved thermal aging of carbon fiber cross-ply laminate $\left[0 / 90_{2}\right]_{\mathrm{s}}$. The curing temperature was $120^{\circ} \mathrm{C}$, so the thermal aging temperature was set to $110^{\circ} \mathrm{C}$. Three sets of samples were used; the reference and two other groups each one corresponding to a time interval; 250 and 400 hours. After the aging, each sample was subjected to tensile loading. The same sample was studied under increasing strain levels and between each step it was examined under optical microscope for transverse cracks. The degradation was also expressed in crack density and the results in terms of average values are presented in Figure 7. 


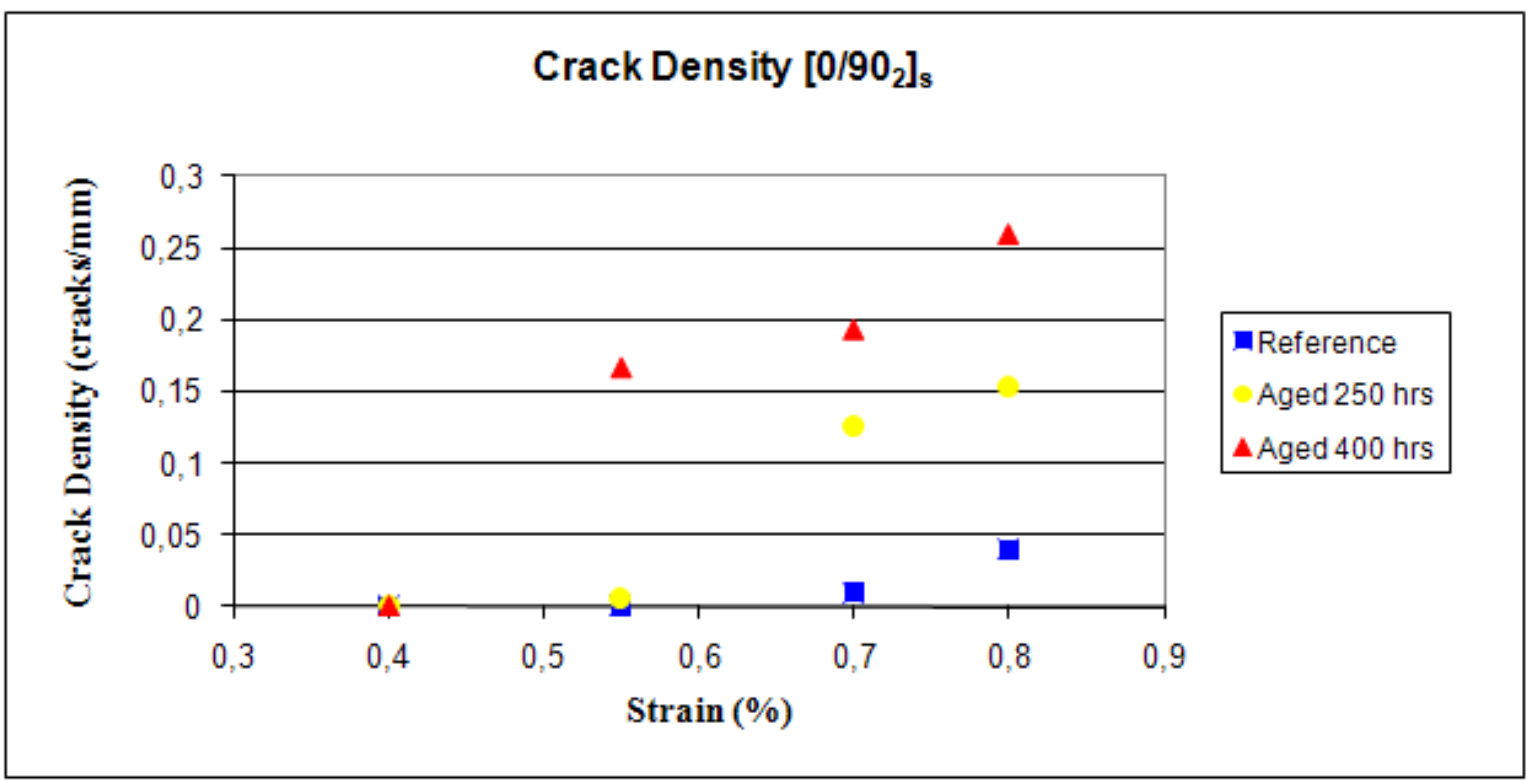

Figure 7: Crack Density dependence on aging time and applied strain

The main conclusion is that the thermal aging does not affect the structural integrity of the material in terms of crack density, when the material is subjected to low strain levels (first cracks appear at about the same applied strain). The increase of the stress introduces cracks, the number of which increases with elevating strain level. In addition, the crack density is a clear function of aging, indicating the above mentioned matrix and interface degradation over time.

\section{Conclusions}

The effect of mechanical and thermal fatigue and thermal aging on CF/EP composites was investigated in the present research. The composite plates were subjected to an elevating number of thermal and mechanical fatigue cycles. For each number of cycles, the number of developed cracks was measured and the crack density was calculated. In addition the effect of chemical shrinkage was estimated by subjecting an unbalanced composite plate to elevating temperatures and measuring the curvature at each step. It is shown that for all test cases the source of degradation (thermal fatigue, mechanical fatigue, thermal aging) has introduced cracks in the transverse layer. 
5th International EEIGM/AMASE/FORGEMAT Conference on Advanced Materials Research IOP Publishing IOP Conf. Series: Materials Science and Engineering 5 (2009) 012020 doi:10.1088/1757-899X/5/1/012020

\section{References}

1. Bechel V T, Camping J D and Kim R Y, "Cryogenic/elevated temperature cycling induced leakage path in PMCs," Composites: Part B: Engineering, Vol. 36, (2005), 171-182

2. Lundmark P and Varna J, "Constitutive relationships for laminates with ply cracks in in-plane loading", International Journal of Damage Mechanics (2005), 14 (3), 235-261

3. Lundmark P and J Varna, Crack face sliding effect on stiffness of laminates with ply cracks, Composites Science and Technology, 66, (2006), 1444-1454

4. C M Lawrence $\mathrm{Wu}$, Thermal and mechanical fatigue analysis of CFRP laminates, Composite Structures, 25, (1993), 339-344

5. Keiichiro Tohgo, Shuji Nakagawa and Kazuro Kageyama, Fatigue behavior of CFRP crossply laminates under on-axis and off-axis cyclic loading, International Journal of Fatigue 28 (2006), 1254-1262

6. David Leveque, Anne Schieffer, Anne Mavel and Jean-Francois Maire, Analysis of how thermal aging affects the long-term mechanical behavior and strength of polymer-matrix composites, Composites Science and Technology 65 (2005), 395-401

7. Varna J, Quantification of damage and evolution modeling in multidirectional laminates, Proc. of the $27^{\text {th }}$ RISÖ International symposium on material science, Roskilde, Denmark, 2006, 349-356

\section{Acknowledgements}

The authors would like to acknowledge the contribution of Dr Roberts Joffe, Derek Ruyle, Nathan Van Velson, Thomas Boulet and Rémi Souchon for their contribution to the present paper. 